\title{
L'imaginaire de la communication à travers le concept de parole en Afrique et en Occident
}

Kouméalo Anate

\section{OpenEdition}

1 Journals

Édition électronique

URL : http://journals.openedition.org/communicationorganisation/2773

DOI : 10.4000/communicationorganisation. 2773

ISSN : 1775-3546

Éditeur

Presses universitaires de Bordeaux

Édition imprimée

Date de publication : 1 novembre 2002

ISSN : 1168-5549

Référence électronique

Kouméalo Anate, «L'imaginaire de la communication à travers le concept de parole en Afrique et en Occident », Communication et organisation [En ligne], 22 | 2002, mis en ligne le 27 mars 2012, consulté le 21 décembre 2020. URL : http://journals.openedition.org/communicationorganisation/2773 ; DOI : https://doi.org/10.4000/communicationorganisation.2773

Ce document a été généré automatiquement le 21 décembre 2020.

(c) Presses universitaires de Bordeaux 


\title{
L'imaginaire de la communication à travers le concept de parole en Afrique et en Occident
}

\author{
Kouméalo Anate
}

1 «Au commencement était le Verbe», d'après l'Evangile selon saint Jean. Au commencement était le Nommo d'après les Dogons du Mali. A l'origine de la parole il y a donc le mythe, il y a l'imaginaire. En fait, l'idée de parole s'exprime à travers des configurations imaginaires et théoriques très variées qui présentent des avatars différents selon les époques, les contextes socioculturels et les paysages conceptuels. Par ailleurs elle a toujours constitué un élément structurant de l'univers cognitif et communicationnel des sociétés. Cependant la parole suscite dans le champ du savoir des représentations changeantes qui oscillent entre un imaginaire éthico-religieux et un imaginaire technico-scientifique.

2 Dans l'épistémè occidentale, notamment à partir du Moyen Âge chrétien, l'inscription sociale de la parole s'est faite sous l'emprise de l'imaginaire religieux, du moins jusqu'à la Renaissance, dont on connaît parallèlement la dimension prométhéenne et anthropocentrique. Après cette période, le rapport à la parole s'est modifié, sous l'influence d'un imaginaire scientifique et technocentré. Dans le même temps l'imaginaire. « la folle du logis ». devenait suspecte, à la lumière d'une certaine rationalité qui s'est imposée au cours de la période identifiée comme «l'âge classique » par Michel Foucault dans Les Mots et les Choses (1966). D'un autre côté, les constructions imaginaires et les images mythiques imprègnent également le domaine scientifique comme l'ont montré certains travaux ${ }^{1}$ sur l'invention scientifique ou sur les innovations technologiques.

Un travail comparatif sur la parole en Occident et en Afrique nous permet de comprendre comment ce concept induit et véhicule un imaginaire spécifique selon les contextes culturels. Au-delà des différences contextuelles, on retrouve des points communs, notamment l'univers mythico-religieux que l'on associe à la parole dans toutes les civilisations. Une observation attentive du champ symbolique et sémiologique de ce 
concept permettrait de parcourir un nouvel espace cognitif en matière de communication. Tout en nous appuyant sur le kabyè (langue du Nord Togo) et le français, pour des raisons de commodité, nous étendrons nos considérations à l'ensemble de l'Afrique et au monde occidental.

\section{La parole dans l'imaginaire conceptuel occidental}

4 Dans le cadre de la langue française la notion de parole traverse des territoires sémantiques très variés: verbe, logos, discours, langage. Au-delà de cette variété de termes, il faut souligner que chacun de ces concepts véhicule un imaginaire particulier.

La notion de verbe évoque un aspect primordial, mystique et démiurgique comme l'illustrent les premiers versets de l'Evangile de St Jean : «Au commencement était le Verbe, le Verbe était auprès de Dieu et le Verbe était Dieu ».

6 Souvent en concurrence avec le terme verbe, le concept de parole a fini par s'en distinguer et recouvrir un champ sémantique autre, le plus souvent caractérisé par une dimension éthico-morale comme le montrent les expressions suivantes: parole d'honneur, donner sa parole, tenir parole. La parole investit aussi un espace juridique et démocratique : le droit à la parole, la prise de parole.

7 Quant au logos, chargé d'un héritage conceptuel grec, il se réfère (dans le champ cognitif français) à la parole maîtrisée, au savoir codifié; il s'inscrit naturellement dans le domaine de la raison, de la philosophie et de la science conceptualisée. D'ailleurs le suffixe logie dérivé de logos, évoque un savoir théorique contrairement au suffixe graphie souvent plus descriptif : ethnologie/ethnographie.

8 En ce qui concerne le terme discours, il désigne une parole plus technique et plus instrumentalisée que l'on retrouve souvent dans le domaine de la politique, de l'idéologie, de la publicité, des sciences du langage et dans le langage scientifique.

9 Le Dictionnaire de linguistique et des sciences du langage, publié sous la direction de Jean Dubois précise que la "parole» a longtemps été confondue avec le langage. Michel Foucault (Les mots et les choses, 1966) montre que dans le contexte occidental, le langage est souverain à l'âge classique, car les mots «représentent la pensée qui elle-même est déjà parole». Les mots sont donc ce qu'ils disent. Le langage n'est pas que communication, il est aussi la pensée. Mais le langage, toujours selon Foucault, finit par se transformer en discours, en l'occurrence au Siècle des Lumières où l'on assiste à une " rupture épistémologique » qui s'accompagne d'une " démythification des mots ». C'est dire que la perception de la parole et le rapport que les hommes entretiennent avec elle changent avec le temps. On note ainsi un déplacement de sens dans l'usage des mots relevant du champ lexical de la parole. Les mots ont progressivement perdu une grande partie de leur dimension symbolique et spirituelle, au profit d'un logos « plus rationnel ».

Il est important de souligner que, dans le cas du français, la plurinomination à propos du concept de parole reflète un héritage gréco-latin, et une histoire de la pensée particulière à l'Occident. Mais cette abondance lexicale constitue-t-elle une richesse ou une pauvreté ? Quelles en sont les conséquences sur le traitement de la parole et sur la vision sociale qui en découle ? L'histoire de la pensée en Occident, telle qu'elle est développée en particulier dans Les Mots et les choses, met en évidence la dissociation progressive qui s'est opérée entre philosophie, savoir et éthique à partir de la Renaissance jusqu'à l'époque contemporaine. Il semble finalement que ce qui apparaissait comme un simple 
phénomène de plurinomination (de la parole) devient en réalité un processus de dissémination de sens.

11 Pour mieux illustrer notre propos nous avons choisi de présenter l'horizon sémantique de la parole sous forme de tableau à quatre entrées représentées par des ensembles signifiants dont chacun recouvre un domaine relativement circonscrit: verbe, parole, logos, discours.

Horizonsémantique de la parole en français

\begin{tabular}{|c|c|c|c|}
\hline Verbe & $\begin{array}{r}\text { Parole } \\
\text { (parabole) }\end{array}$ & $\begin{array}{l}\text {-logie } \\
\text {-logique }\end{array}$ & $\begin{array}{l}\text { Discours } \\
\text { (construction) }\end{array}$ \\
\hline \begin{tabular}{l}
\multicolumn{1}{c}{ aspect } \\
primordial \\
(énergie \\
transformatrice): \\
au \\
commencement \\
était le verbe \\
- puissance \\
créatrice et \\
démiurgique : \\
Dieu crée par la \\
parole (genèse) \\
- dynamisme \\
mystique \\
- réalité magico- \\
spirituelle \\
- dimension \\
cosmique
\end{tabular} & $\begin{array}{l}\quad \text { immersion } \\
\text { dans le social } \\
\text { - projet de } \\
\text { persuasion, } \\
\text { mobilisation } \\
\text { - dimension } \\
\text { éthico-morale } \\
\text { - capacité } \\
\text { réparatrice } \\
\text { - similitude } \\
\text { parole donnée, } \\
\text { parole tenue, } \\
\text { parole } \\
\text { d'honneur, } \\
\text { prise de parole, } \\
\text { droit de parole, } \\
\text { parole de Dieu }\end{array}$ & $\begin{array}{l}\text { Parole maîtrisée, } \\
\text { destinée à maîtriser } \\
\text { le monde } \\
\text { - codification } \\
\text { - philosophie } \\
\text { - logique } \\
\text { - pensée/raison } \\
\text { - savoir }\end{array}$ & $\begin{array}{l}\quad \text { Parole } \\
\text { instrumentalisée } \\
\text { - politique } \\
\text { - idéologie } \\
\text { - religion } \\
\text { - performance } \\
\text { - publicité } \\
\text { - discours } \\
\text { scientifique } \\
\text { - positivisme }\end{array}$ \\
\hline
\end{tabular}

12 Au regard du contenu de ce tableau il semble désormais difficile de regrouper les catégories Verbe, Parole, Logos, Discours sur le même axe paradigmatique. Ces catégories ressortissent désormais à des registres différents. Ainsi Verbe s'inscrit principalement dans le domaine religieux. Parole évoque davantage le domaine social. Logos se veut une parole rationalisée et de démonstration à visée scientifique, enfin Discours comporte une dimension idéologique et se caractérise par une dynamique d'instrumentalisation.

En résumé, à propos du concept de parole, la langue française ne se contente pas d'un simple emprunt à différentes sources étymologiques greco-latines; elle donne lieu à des déplacements de sens et à un redéploiement sémantique. Rappelons qu'en grec un seul vocable exprime généralement les idées de parole, discours, causes, mode d'expression: logos. Mais dans le cadre de la gestion sémantique du français, le terme logos finit par être dissocié des notions de parole et de discours, pour être intégré à de nouvelles association de sens.

\section{La parole dans l'imaginaire conceptuel africain}

Contrairement à la variété d'avatars de la parole en français, on constate que dans la plupart des langues africaines, il n'existe qu'un seul vocable pour dire l'ensemble parole- 
langage-logos-discours. Le mot kabyè pour traduire tous ces termes, est tom. C'est le cas dans de nombreuses langues africaines, et dans le créole des Caraïbes tel que l'indique le sens du mot pawol en créole. Cependant cette désignation apparemment réduite s'accompagne d'une très forte polysémie. À ce propos, Paul Ricoeur (La métaphore vive. Seuil. 1975) souligne que la polysémie n'est nullement synonyme de pauvreté : elle témoigne plutôt « du caractère ouvert de la texture d'un mot ».

Prenons à titre d'exemple ces expressions kabyès dans lesquels nous retrouvons le mot parole, tom

Tom héyu = (le fait de) dire la parole

Tom yodu = (le fait de) parler la parole (discussion, échange)

Tom kalu = (le fait de) lire la parole

Tom mawu (le fait de) dessiner ou d'écrire la parole

Tom huw = juger la parole (rendre justice, instruire un procès)

Tom suzu = (le fait d'annoncer, de diffuser la parole)

Tchanawtatom = parole des pères, parole des Anciens ( = tradition, histoire)

«La parole ne pourrit jamais» disent de nombreux proverbes africains ${ }^{2}$. Cette phrase illustre une certaine conception de la parole perçue comme élément durable et fiable. L'idée de pérennité de la parole est bien présente dans l'une des métaphores qui la représente le mieux, à savoir la "graine ». On retrouve l'expression "graine de parole " ${ }^{3}$ (parfois «arbre de parole») dans la plupart des langues africaines. Ainsi la parole dans cet imaginaire, a non seulement une consistance solide mais elle est surtout synonyme de germination, de fécondité, de naissance ou de création permanente. Le texte transcrit par le professeur Sory Camara (université de Bordeaux II) qui a fait de nombreuses recherches sur la parole chez les peuples mandingues, illustre bien cette conception africaine de la parole :

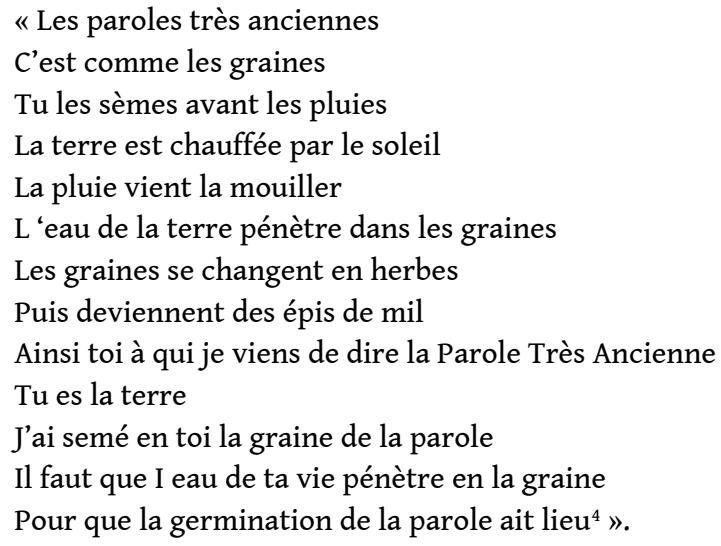

24 Cette parole s'inscrit naturellement dans un imaginaire holistique. À ce propos, le chercheur congolais, Pius Ngandu Nkashama propose une vision globale de la parole à travers une série de trois articles qu'il a publiés ${ }^{5}$ sur la parole africaine. Ses travaux et sa démarche herméneutique lui ont permis de construire une théorie de la parole, qui est celle de la totalité: la parole comme totalité créatrice, structurante, relationnelle, ouverte sur la connaissance du monde. Il établit également différentes distinctions ayant trait à la parole : parole cosmique, parole mythologique, parole symbolique, parole linguistique.

Selon Pius Ngandu Nkashama. dont nous reproduisons quelques conclusions ci-dessous, la parole africaine présente les caractéristiques multiples : 

elle-même dans une totalité cosmique, non pas comme métaphore mais comme un « langage intégral », un mode d'appréhension, d'exploration et de compréhension du réel. Elle est aussi " considérée comme faisant partie intégrante de l'individu, comme élément essentiel, non seulement de l'ordre de la connaissance mais souvent même de l'ordre de l'existence ${ }^{7}$ ».

- Elle est valeur et action. Elle organise le monde, elle agit sur les éléments de la nature (comme faire tomber la pluie), elle déchiffre le monde. Cette parole " possède une existence propre indépendamment de l'individu qui la profere $»^{8}$.

28

La parole est aussi questionnement du monde, donc savoir. Elle constitue une " porte » qui permet l'accès à la fois au monde intérieur (connaissance de soi), et au monde extérieur (connaissance du monde). Pius Ngandu précise que c'est de cette « connaissance du monde » que dérive également la « parole de la science ».

Finalement, la dimension globalisante de la parole africaine, ainsi que celle qu'on observe dans la tradition judéo-chrétienne, se démarquent de la pensée occidentale actuelle qui ne « réalise plus sa relation au cosmos»; pourtant «le monde se lit constamment sur le mode mythique ou mythologique ", remarque Pius Ngandu. Cependant il nous semble que si l'Occident moderne a fini par exclure de ses modèles sociaux et intellectuels la dimension symbolique et mythique de la parole, il n'en a pas toujours été ainsi, si on considère l'héritage laissé par la tradition judéo-chrétienne.

\section{Imaginaire africain et judéo-chrétien}

30 À l'image des langues africaines, l'hébreu privilégie un seul terme pour les différentes acceptions de parole : davar. On peut par ailleurs établir un parallèle entre l'imaginaire de la parole dans les deux contextes culturels : africain et judéo-chrétien, mais revenons d'abord à la mythologie de la parole en milieu africain. Nous avons choisi la mythologie dogon parce qu'elle est l'une des plus complexes et des plus représentatives de l'univers mythique africain connues jusqu'à ce jour.

31 En effet selon la cosmogonie des Dogons du Mali rapportée dans Dieu d'eau (1966) de Marcel Griaule. la Terre est un corps, et ce corps est femme (épouse de Dieu, Amma), et Dieu s'unit à sa femme. L'eau, semence divine, pénétra au sein de la terre. Il en résulta deux êtres, deux produits homogènes de Dieu, d'essence divine. Ce couple de jumeaux est le Nommo. Il s'agit d'un couple complet et parfait par ses huit membres. Son chiffre 8 est le symbole de la parole. Et le Nommo est parole.

32 Ce récit dogon rappelle incontestablement le récit biblique concernant l'incarnation de Jésus, Fils de Dieu, né d'une femme. Il est présenté comme la parole originelle qui se fait chair et devient homme afin d'apporter aux hommes la vie de Dieu, la parole de Dieu. Le Christ représente donc la parole matérialisée.

Finalement on peut observer une certaine homologie structurale entre les deux récits, à travers les figures du Christ-parole et du Nommo-parole. Ici encore nous aurons recours à un tableau récapitulatif et comparatif à quatre entrées : le domaine chrétien et africain d'où nous partons pour comprendre l'identification de la parole, ses représentations ainsi que ses fonctions. 


\begin{tabular}{|c|c|c|c|}
\hline Domaine & Identification & Représentations & Fonctions \\
\hline \multirow[t]{2}{*}{ Chrétien } & Dieu & Père & créateur \\
\hline & Christ & $\begin{aligned} & \text { Parole/Fils } \\
& \text { - transformateur } \text { - réparateur } \\
& \text { - rédempteur } \\
& \text { - pédagogue }\end{aligned}$ & $\begin{array}{l}\quad \text { Parole } \\
\text { - créatrice } \\
\text { - révélatrice } \\
\text { - réparatrice } \\
\text { - unificatrice } \\
\text { - éducatrice } \\
\quad \text { Sagesse } \\
\text { (proverbes, } \\
\text { parabole) } \\
\quad \text { connaissance }\end{array}$ \\
\hline \multirow{2}{*}{$\begin{array}{l}\text { Dogon } \\
\text { (africain) }\end{array}$} & Amma & Père & créateur \\
\hline & Nommo & $\begin{array}{l}\quad \text { Parole/Fils } \\
\text {-transformateur } \\
\text { - réparateur } \\
\text { - rédempteur } \\
\text { - pédagogue } \\
\text { - Ingénieur }\end{array}$ & $\begin{array}{l}\quad \text { Parole } \\
\text { - créatrice } \\
\text { - révélatrice } \\
\text { - réparatrice } \\
\text { - unificatrice } \\
\text { - éducatrice } \\
\quad \text { Sagesse } \\
\text { (proverbes, } \\
\text { parabole) } \\
\text { connaissance }\end{array}$ \\
\hline
\end{tabular}
intemporel de la parole. Elle est parole des origines (créatrice, structurante, ordonnatrice), et parole de l'accomplissement: alpha et oméga. L'une des idées fondamentales que nous retiendrons ici est que ceux qui reçoivent cette parole finissent par devenir parole eux-mêmes. Les huit ancêtres primordiaux, dans la mythologie des Dogons. créés avec de l'argile et auxquels le Nommo (fils du Dieu Amma) a donné la parole, deviennent eux aussi des Nommo en réintégrant le sein de la Terre mère ; à leur tour ils enseignent la parole aux hommes. Et l'homme peut lui aussi devenir parole.

\section{Différentiation et dissémination de sens : logos- discours}

Dans le cas de la langue française la dissémination sémantique résultant de la différentiation des acceptions de la parole a généralement privilégié les domaines à caractère scientifique. À l'opposé, l'un des aspects de la parole qui n'obéit pas au principe 
d'économie est désigné par le terme palabre. Il nous semble que, dans la nouvelle épistémè occidentale, il y a un rétrécissement du territoire sémantique de la parole ou une mise à l'écart de celle-ci. Elle n'apparaît plus comme un concept opératoire à utiliser dans une démarche méthodologique, même si en temps qu'objet d'étude elle a ressurgi dans le champ d'observation de la communication (sous une acception limitée). Cet ostracisme qu'elle a subi est probablement dû au fait qu'elle dégage un "parfum » d'émotivité, contraire à l'objectivité distanciée généralement associée à la raison.

En réalité la pensée rationaliste issue de la pensée grecque, s'est confortée à la Renaissance, renforcée au siècle des Lumières et enrichie d'une légitimation scientifique par le positivisme à partir du xix ${ }^{e}$ siècle. C'est dans ce sens que Jacques Derrida dénonce le logo centrisme de la philosophie occidentale (De la Grammatologie, 1967). L'Occident a fait du logos l'origine de la vérité. Le logos s'inscrit alors dans la supériorité d'une parole construite qu'on assimile à la vérité. Il s'agit dans ce cas d'une parole maîtrisée, et destinée à maîtriser le monde. Cette conception du logos a favorisé l'émergence de deux états d'esprit corollaires d'un imaginaire spécifique : la toute-puissance de la science et celle de la raison. Au nom d'une exigence de codification fiable, le discours scientifique exclut tout ce qui est suspecté d'irrationnel, et d'informel. D'où la vision péjorative de la " palabre » africaine, associée à une perte ou à un gaspillage de temps, c'est-à-dire à une très mauvaise gestion de la communication : les palabres interminables. Or la "palabre » dans le contexte culturel africain n'est autre chose que la parole se matérialisant dans sa fonction sociale et juridique.

\section{La palabre introuvable en Afrique}

L'imaginaire de la parole tel qu'il s'est élaboré dans le paysage conceptuel occidental s'est répandu, à la suite des expansions coloniales, dans d'autres aires culturelles, notamment en Afrique. Toujours dans le cadre de l'épistémè occidentale, la parole a subi une dévalorisation en termes de prestige rationnel, et a donc fini par être reléguée au bas de l'échelle scientifique, en étant associée à des conceptualisations à tendances péjoratives. Tel est le cas par exemple du mot palabra, qui signifie « parole » en espagnol, utilisé pour désigner certains exercices de la parole en Afrique.

À l'origine palabre désignait le présent fait à un roi noir des côtes d'Afrique pour se concilier ses bonnes grâces. Par extension ce mot fut utilisé pour désigner les pourparlers à l'occasion de la remise de ces présents. Il est important de constater que dès ce moment le mot palabre a été chargé d'un sens très péjoratif, devenu le sens courant du mot en Occident. La palabre est alors définie comme un discours vain et inutile, une discussion interminable et oiseuse. Cette réorientation sémantique du mot espagnol fait que, non seulement la parole a été détournée de son sens, mais a polarisé un transfert de sens négatif pénalisant une pratique culturelle très mal connue de ceux qui l'ont cataloguée.

41 Pour réhabiliter la palabre africaine, il faudrait revenir au sens originel de palabra en espagnol qui est neutre. Cela correspondrait mieux à ce que l'on retrouve dans la plupart des langues africaines. Par exemple en kabyè, les expressions pour dire "tenir une palabre » c'est "parler la parole» (tom yodu) lorsqu'il s'agit de conversation ou de discussion pour prendre une décision importante. On trouve dans la langue mina ${ }^{9}$ une expression équivalente, alegnakpo. qui signifie littéralement «tenir, prendre, saisir la 
parole ». Il est question aussi de « juger la parole » (tom huw) lorsqu'il s'agit d'instruire un procès, de rendre justice.

Il existe en fait plusieurs sortes de "palabres ». Le philosophe camerounais Jean-Godefroy Bidima (in La palabre: une juridiction de la parole. Michalon. 1997) les regroupe en deux catégories : les "palabres iréniques ». tenues en dehors de tout conflit (à l'occasion d'un mariage, deuil, vente, etc.), et les "palabres agonistiques" qui interviennent dans la gestion de conflits. Selon la nature de la "palabre» toute la communauté peut être convoquée. Dans d'autre cas la séance est limitée au conseil de sages, conseil de famille, etc. La " palabre" a ses palais, ses tribunaux : traditionnellement on se réunissait sous l'arbre, ou dans le vestibule de la maison royale. Mais la "palabre " peut se tenir dans tout autre lieu (maison, entreprise, etc.). Cette pluralité de lieux montre qu'il n'est donc pas toujours question " d'arbre à palabres ».

La « palabre » comme art social, participe à l'art de bien communiquer et de «faire tenir ensemble " la société, le groupe. Il fonctionne sur plusieurs principes: le principe de dialogue négociation, la recherche de la paix, l'harmonie du groupe, la réparation par le don. la mémoire des référents socioculturels.

Dans l'imaginaire véhiculé par la "palabre ", aucune organisation n’est isolée, séparée des préoccupations sociales et des représentations de l'idée de lien social et du bien être de tous. Une organisation est une partie d'un tout (la communauté). Avant toute chose il s'agit d'un espace de rencontre dans la " parole donnée ». dans la reconnaissance de soi et de l'autre, dans la recherche de solutions communes. C'est ce qui « cimentait autrefois le vivre-ensemble». selon G. Bidima. Et c'est dans ce cadre que la discussion, et la négociation sont possibles. À l'instar des entreprises japonaises qui pratiquent une sorte de "palabre » permanente, «ringesei », afin de mettre en œuvre dans l'entreprise un consensus provisoire de coopération et une interaction négociée et temporaire, la " palabre » africaine pourrait apporter davantage à l'entreprise. Grâce à la technique de la «palabre » on peut régler des conflits latents dans diverses situations avant que la crise n'éclate. On peut associer le plus grand nombre de participants à la prise de décision: cette implication plus grande renforce le sens de la responsabilité. - «réparation partagée » : faire en sorte que celui qui a tort ne se sente pas humilié à la fin. Cela permet de sauver l'harmonie du groupe, car la «la palabre institue un espace public de discussion... Elle institue une médiation symbolique à plusieurs entrées. Avec la palabre nous quittons l'immédiateté des relations pour entrer dans un réseau de médiations qui s'emboîtent à l'infini. Dans cette mise en abyme, chaque médiation débouche sur une autre médiation qui, elle-même, en engendre de nouvelles. ${ }^{10}$ Nous n'allons pas nous attarder sur le fonctionnement et le rôle de la «palabre ». car le propos de l'article n'est pas l'analyse des fonctions de la parole. Toutefois, qu'on l'appelle palabra (en espagnol), tom (en kabyè), davar (en hébreu) ou logos (en grec), la parole semble intrinsèquement liée à la technique dès l'origine. Les concepts de parole et technique sont en fin de compte indissociables et partagent un même espace symbolique.

\section{Parole et technique : de la jupe tressée à la toile mondiale}

Le rapport entre parole et technique peut se lire au niveau du traitement de la parole par l'usage soit des outils de communication divers (tam-tam, radio, télévision, téléphone, 
etc.) soit, dans le cas du traitement automatique, par des techniques de l'intelligence artificielle.

En restant dans le domaine des représentations et de l'imaginaire social nous remarquons que la parole et la technique ont toujours suscité chez l'homme un rapport mythicoreligieux. Parole et technique sont généralement perçues comme ayant une origine divine. En effet dans la mythologie dogon du Mali mentionnée dans ce travail, l'homme reçoit la parole en même temps que la technique, puisque que l'objet «technique » sert de support au don de la parole. Le Nommo-ingénieur transmet un savoir technique en même temps qu'il donne la parole (jupe tressée, le tissage, le grenier, le tam-tam...). Ainsi dans l'imaginaire dogon. la parole va de pair avec le développement technique et avec la connaissance. Les outils techniques sont faits de parole et transmettent un enseignement. L'objet ou l'outil parle pourrait-on dire. Ainsi la première parole fut transmise à l'homme par le Nommo à travers les fibres d'une jupe tressée, la deuxième parole à travers le métier à tisser, la troisième à travers tam-tams et tambours. La parole tressée puis tissée (de ce fait on pourrait parler de parole visuelle), devient son et vibration.

47 La métaphore de la "parole tissée » renvoie indubitablement à l'idée de la parole comme « texte » et comme « toile », ce qui nous plonge dans l'imaginaire moderne des nouvelles technologies de communication dont Internet est le prototype. N'oublions pas que les mots texte et toile viennent d'un même mot latin "texere" qui signifie tisser. La métaphore de "toile d'araignée» qui désigne Internet fait allusion aux réseaux d'ordinateurs interconnectés de part le monde formant une « toile mondiale ». Ainsi cette possibilité d'une toile mondiale, d'une parole tissée à l'échelle mondiale contient déjà le rêve d'absolu, le mythe d'une société où tout communique et se communique dans une harmonie totale. Ce rêve messianique d'une société de communication que suscitent certaines innovations techniques comme l'avènement de l'informatique avec ses extensions donne l'illusion d'une maitrise totale de la connaissance grâce à la sophistication de la technique et de l'intelligence artificielle maîtrisées par l'intelligence scientifique humaine. Ce rêve d'absolu se trouve cristallisé actuellement autour d'Internet, médias a fonctionnement holistique, auquel l'on voue un véritable culte comme l'a si bien analysé Philippe Breton dans Le culte de l'Internet (2000). La technique, de manière générale, permet le rêve, rêve de perfectionnement et d'absolu.

En somme, la conception mythique de l'origine de la parole et de la technique ainsi que le rapport mystique et religieux que nous entretenons souvent avec certains outils techniques sont des phénomènes permanents. Ces deux phénomènes ne relèvent nullement d'un passé révolu ; ils ne sont pas non plus le produit exclusif de la modernité. Ils se manifestent avec une étonnante récurrence à travers les époques et les différentes civilisations. Certes les génies ingénieurs ne sont plus les Nommo, ils sont remplacés aujourd'hui par nos ingénieurs informaticiens (de la high technology) qui sont perçus dans notre imaginaire comme des sorciers ou des démiurges.

\section{Conclusion}

49 L'imaginaire de la communication observé à partir des différentes déclinaisons du concept de parole demeure principalement religieux, quelles que soient les civilisations et les époques. L'Occident, malgré le détour par la "rationalité scientifique », n’a pas réussi à échapper à l'univers mythicoreligieux dans lequel nous introduisent constamment parole et technique. Cependant il y a un absent : Dieu. Dieu était présent dans la première 
circularité qui unissait l'homme à la parole et à la technique. Au fil des innovations technologiques et scientifiques, l'homme-ingénieur tend à se substituer à Dieu. Une nouvelle circularité apparaît dans les termes suivants: homme $\rightarrow$ technique $\rightarrow$ homme (et/ou société). Pourtant la vision téléologique omniprésente repose non plus sur la parole mais surtout sur la technique, comme si à travers l'homme la parole s'était définitivement «mécanisée » ou avait été réduite, par confusion, à la technique. Dès lors il est permis d'attendre d'une technique comme Internet qu'elle refasse le lien social, et préside à l'avènement d'une société de communication. On n'hésite plus à identifier Internet à la société tout entière, lorsqu'on parle de société de l'information et de la communication. Or la technique reste un moyen (ou un ensemble de moyens) au service de la parole, au service de l'homme et de la société. Par ailleurs la parole possède une dimension ontologique. Elle nous constitue, nous lie et nous transcende en même temps. Cette réalité protéiforme de la parole garde encore une dimension insaisissable pour l'homme malgré les techniques de plus en plus sophistiquées qui permettent un meilleur traitement et une meilleure circulation de la parole.

\section{BIBLIOGRAPHIE}

BIDIMA, J.-G., La Palabre, une juridiction de la parole. Paris : Michalon. 1997. 127 p.

BRETON, P.. La Parole manipulée. Paris, Découverte. 1997. 220 p.

BRETON, P., Le Culte de l'Internet : Une menace pour le lien social ? Paris : Découverte, 2000.126 p.

DERRIDA, J.. De la Grammatologie. Paris : Ed. de Minuit. 1967. DURAND. G., L'Imaginaire : Essai sur les sciences et la philosophie de l'image. Paris : Hatier. 1994. 80 p.

ELLUL. J., La Parole humiliée. Paris : Seuil. 1981. 304 p.

FOUCAULT, M., Les Mots et les choses. Archéologie des sciences humaines. Paris : Gallimard. 1990. $400 \mathrm{p}$.

GRIAULE, M.. Dieu d'eau : Entretiens avec Ogotemmêli. Paris : Fayard, 1966. 233 p.

GUSDORF, G., La Parole. Paris : PUF. 10e édition, 1988.

NDONGO, J. F.. Un regard africain sur la communication. Yaoundé: Editions St Paul. 1996. 105 p.

PACERE, F. T.. Le Langage des tam-tams et des masques en Afrique. Paris : L'Harmattan. 1992. 350 p.

\section{Articles et périodiques}

Cahiers ethnologiques. Histoires et Cultures : Palabres autours des paroles de Sory Camara. Presses universitaires de Bordeaux. 1998. $\mathrm{n}^{\circ} 19$.

NGANDU. N. P., Parole africaine et métaphore cosmique. Cahiers des religions africaines, janvier 1979. n' 25.

Sciences Humaines : l'imaginaire contemporain, janvier 1999. $\mathrm{n}^{\circ} 9$. 


\section{NOTES}

1. Cf Gilbert Durand. Introduction à la Mythodologie. Mythes et Sociétés. Paris. L.G.F.-Biblio Essais. 2000. Gérard Holton. L'imagination scientifique Paris. Gallimard, 1981. Victor Scardigli, Les sens de la technique. Paris. PUF, 1992.

2. Cf le proverbe kabiyè : Tom i buki

3. Tombié en kabyè ou xókwín en Fon (au Bénin)

4. Sory Camara in Cahiers Ethnologiques. Histoires et cultures: palabres autour des paroles de Sory Camara. p. 110

5. Pius Ngandu Nkashama. Parole africaine et métaphore cosmique, in Cahiers des religions africaines, vol. 13. $\mathrm{n}^{\circ}$ 25. Janvier 1979

6. Pius Ngandu Nkashama. op. cit. p. 77

7. Pius Ngandu Nkashama. op. cit. p. 49

8. Pius Ngandu Nkashama. op. cit. p. 55-56

9. Le mima est une des langues les plus parlées au sud du Togo

10. Jean-Godefroy, Bidima. op. cit. p. 13

\section{RÉSUMÉS}

L'imaginaire de la communication est indissociable de celui de la parole et de la technique qui l'accompagne. Cependant chaque aire civilisationnelle a tissé des rapports spécifiques entre techniques, parole et imaginaire, comme le montre une approche comparée des imaginaires conceptuels occidentaux, judéo-chrétiens, et africains. Or les différents processus de différentiation et de dissémination de sens à propos des concepts parole-logos-discours, ont sans doute masqué les véritables relations entre les discours scientifiques et la prégnance d'un imaginaire mythico-religieux. Et pourtant, au-delà des frontières conceptuelles mouvantes que l'évolution technique redessine en permanence autour de la parole, on constate la récurrence d'un imaginaire mythico-religieux qui précisément transcende les délimitations de domaines de savoir et d'aires civilisationnelles.

The imaginaire of communication is inseparable from that of speech and the technologies contemporary with it. Every civilization has created spécific relationships between technology, speech, and the imaginaire, as is revealed by a comparative approach to modem Western. JudeoChristian, and African symbolic worlds. Now, the various processes by which meaning has been differentiated and dispersed--as in the progressive distinction speech-logos-discours-have probably disguised the real relations between scientific discourses and the mythico-religious world that lias an impact on them. Nevertheless, beyond moving conceptual frontiers, constantly re-dejined by technical evolution, we discover that a mythico-religious imaginaire remains, and that it transcends civilizations and domains of knowledge. 
INDEX

Mots-clés : imaginaire de la communication, parole, mythe, logos, technique

\section{AUTEUR}

\section{KOUMÉALO ANATE}

Kouméalo Anaté termine actuellement un doctorat en Sciences de l'Information et de la Communication à l'Université de Bordeaux 3. Son travail de thèse traite des usages et de l'imaginaire d'Internet en France et au Togo. Quelques axes de recherche : Etude des médias : logique technique et logique communicalionnelle. usages et imaginaire des nouvelles technologies, E-learning. Nouvelles technologies éducatives et coopération Nord/Sud (cas des universités virtuelles en Afrique) : mondialisation et marchandisation de l'éducation et Médias et littérature. Usages des médias dans les littératures négroafricaine 